\title{
Intraoperative Problems and Management in Geriatric Trauma Patients
}

\author{
Iztok Potocnik* and Vesna Novak-Jankovic \\ Clinical Department of Anaesthesiology and Intensive Care Medicine, University Medical Centre Ljubljana, Slovenia
}

Received: 制 June10, 2018; Published: 制 July 16, 2018

*Corresponding author: Iztok Potocnik, Clinical Department of Anaesthesiology and Intensive Care Medicine, University Medical Centre Ljubljana, Slovenia

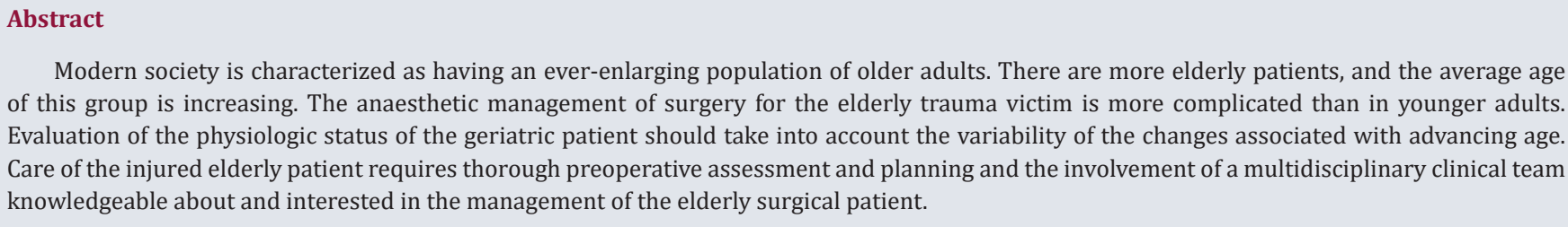

Modern society is characterized as having an ever-enlarging population of older adults. There are more elderly patients, and the average age of this group is increasing. The anaesthetic management of surgery for the elderly trauma victim is more complicated than in younger adults. Evaluation of the physiologic status of the geriatric patient should take into account the variability of the changes associated with advancing age. Care of the injured elderly patient requires thorough preoperative assessment and planning and the involvement of a multidisciplinary clinical team knowledgeable about and interested in the management of the elderly surgical patient.

\section{Introduction}

Traumatic injury is the four most cause of mortality in elderly patients. At the end of the last century $20 \%$ of injuries occurred in the elderly population. These patients respond differently to trauma, recover more slowly and have higher morbidity and mortality. It is not clear if these differences are due to increased comorbidity or decreased physiologic reserve [1,2]. After traumatic injury, it is important that all interventions be evidence based. There are few prospected randomized trials that focus on elderly issues.

\section{Definition}

There is no uniform definition of the term elderly. Historically, the term elderly was applied to individuals over 65 years of age. However, aging is now viewed as a physiologic continuum rather than chronologic age. Nowadays we divide geriatric population into young old - 65-80 years and oldest old- over 80 years [1].

\section{Aging}

Aging is a universal and progressive physiologic phenomenon characterized by degenerative changes in both the structure and the functional reserve of organs and tissues. Aging is a consequence of free radicals damage within mitochondrial DNA (oxidative stress) [3]. Elderly patients (arbitrarily defined as being over 65 years of age) are vulnerable to the adverse effects of anaesthesia. With advancing age, the autonomic nervous system, heart and blood vessels become less capable of maintaining hemodynamic stability. Increased arterial rigidity and increased sympathetic nervous system activity contribute to the increase in systemic vascular resistance. Increased stiffness of the hypertrophied elderly cardiac ventricle leads to increased end-diastolic pressure with severe diastolic dysfunction. With advancing age, parasympathetic activity decreases while sympathetic neural activity increases. Elderly subjects manifest a reduced responsiveness to beta-adrenergic stimulation and reduced baroreceptor reflex [4].

Changes in the respiratory system with age comprise: decline in compliance of bony thorax, loss of respiratory muscle mass, decrease in alveolar gas exchange surface and decrease in central system responsiveness. Aged lungs have some features of chronic obstructive lung disease, increased residual volume, reduced vital capacity and FEV1 [5]. The target organ for all anesthetic agents is the central nervous system. Aging produces a decrease neural density and loss of $30 \%$ brain mass at the age of 80 years (mostly grey matter). Signs of peripheral nervous system aging are marked by a loss of motor, sensory and autonomic fibers and decrease in conduction velocity. Aging decreases functional capacity of other organ system such as hepatic, renal and endocrine system. Administration of drug in the elderly compared to young subjects results in higher blood levels, due to a smaller volume of distribution and due to a slower drug metabolism. The brain is more sensitive to the drug in the elderly and all these effects conspire to increase the length of time that drug is active in the elderly patient [5].

\section{Functional Reserve}

A decline in the functional reserve may in the elderly patient participate a serious decline in performance when the elderly patient is exposed to stress and increases the risk of age-related disease. The older patient is less able to preserve homeostasis in face of such a physical insult. The variability of this decline is rooted within lifestyle, environmental factors, genetics, and presence of age related disease. Older trauma patients do not cope as well as younger adults. Elderly patients are less able to preserve sufficient 
perfusion of vital organs. After injury, they are more likely to arrive in the emergency department in hypotensive shock and to be hypothermic. The decreased functional reserve contributes to the higher percentage of the geriatric trauma victims that appear in the early trauma mortality statistics. Limited physiologic reserve means that the prognosis of the elderly injured patient is much better when the patient is rapidly transported to a trauma center [1].

In geriatric patients there is reduction of cardiovascular, respiratory, renal and liver functions. There is very little functional reserve (difference between the basal and maximal function of organs) which is safety margin available to the patient during anaesthesia and the postoperative period [5]. Trauma, surgery and administration of anaesthetic drugs often affect cardiovascular physiology to great degree. Randomized studies and a metaanalysis of several randomized clinical trials in non-cardiac surgery patients, comparing outcome with regional and general anaesthetic techniques have shown little evidence of improved outcome and reduced post-operative morbidity and mortality [6]. The importance of management in keeping adequate organ perfusion pressure is often underlined.

\section{Intraoperative Anaesthetic Management}

Anesthesia should be safe with smooth induction, maintenance and quick reversal without producing any cardiovascular, respiratory and nervous complications [5]. To avoid hypotension in the elderly trauma patient, the dose of etomidate, propofol, barbiturates and benzodiazepines need to be reduced. A reduction in the dosage of morphine and other opioids is recommended [1]. Clinically, the doses of neuromuscular blockers are usually unchanged. Aspiration is more common [1]. Multiple retrospective and prospective studies have arrived at the same conclusion. No significant difference in outcome can be attributed solely or predominantly to the use of any specific agent, and no clear and objective benefit can be demonstrated for using regional rather than general anaesthesia [5]. Choice of anaesthesia depends on the patient's general condition, nature of surgical procedure and the experience of the anaesthesiologist. Psychological preparation, appropriate premedication and patient prewarmed is important.

Airway maintenance may be more difficult because of osteoporotic mandibles, temporo-mandibular joint stiffness, loose teeth, cervical spondylosis, ... Maintenance of normothermia is essential because older patients have an impaired ability to sense a colder temperature, less subcutaneousfat, reduced heat generation, reduced ability to vasoconstrict and shiver. A low threshold for early invasive monitoring initiation is used in elderly patients. This is important for the optimization of 02 delivery [1]. It is also important to recognize early patients in whom aggressive additional resuscitation is necessary. There is evidence of the predictive value of the Revised Trauma score, Injury Severity Score (ISS) and Geriatric Trauma Survival Score. The adequacy of resuscitation may be estimated using arterial base deficit [1].

The primary survey in the geriatric patient is no different from that in the younger adult.

\section{Neuraxial Anaesthestic Techniques}

Neuraxial anesthetic techniques include (spinal and epidural blocks) are often used in the trauma surgery. Both techniques can result in sympathetic blockade, resulting in decreases in both preload and afterload and ultimately reducing cardiac output. The decision to use neuraxial anesthesia for the high-risk cardiac patient may be influenced by the dermatomal level of the surgical procedure. Older age is associated with a higher upper level of anaesthesia after epidural administration of local anaesthetic. Higher levels of anaesthesia were attributed to reduced leakage of local anaesthetic because of progressive sclerotic closure of the intervertebral foramina [5]. Epidural blockade that is restricted to the level of the low thoracic and lumbar region (T5-L4) results in a peripheral sympathetic blockade with vascular dilatation in the pelvis and lower limbs and decrease in mean arterial pressure. This decrease is compensated with a reflex increase in efferent sympathetic vasoconstriction above the level of the block; by release of catecholamines from the adrenal medulla [5]. This increased activity may result in increased cardiac contractility and increased heart rate. Reduction in mean arterial pressure during lumbar epidural anaesthesia may increase myocardial ischemia in some patients with coronary artery disease. Activation of the sympathetic nervous system may result in myocardial ischemia and infarction [5]. Atrial fibrillation and tachycardia are common after cardiac and thoracic surgery. Thus, sympathetically mediated decreases in myocardial oxygen supply may be a major factor of postoperative cardiac morbidity [5]. A very useful technique is also thoracic paravertebral blockade (TPB). There is also a potential advantage of avoiding central neuraxial hematoma with this technique. It was presented that TPB can resolve ST segment depression during general anaesthesia and thus is useful in treatment of angina pectoris [7]. The studies which compared TPB with TEA found no difference in analgesia. In the study of Richardson et al. was found that TPB (bupivacaine) was superior in terms of analgesia, pulmonary functions, neuroendocrine stress responses, side effects and postoperative respiratory morbidity compared to TEA (bupivacaine) [8].

\section{Peripheral Nerve Blocks}

Peripheral nerve blocks are used frequently in elderly patients. Sensory and motor blocks last longer in elderly than in younger patients (approximately 2.5 times longer). These alterations are attributed to a decrease in the conduction velocity of the peripheral nerves and to a gradual degeneration of the peripheral nervous system. By the age of 90 years, one third of the myelinated fibers have disappeared from peripheral nerves [9].

\section{Summary}

Modern society is characterized as having a never enlarging population of older adults. There are more elderly patients, and the average age of this group is increasing. The anaesthetic management of surgery for the elderly trauma victim is more complicated than in younger adults. Several factors are essential for obtaining the best outcomes: careful preoperative evaluvation and optimization, minimization of perioperative stress, perioperative attention to 
avoid clinical complications from fluid and electrolyte balance and impaired cardiovascular and respiratory function.

\section{References}

1. Lewis MC, Abouelenin K, Panigua M (2007) Geriatric trauma: Special Considerations in the Anesthetic Management of the Injured Eldery Patient. Anesthesiology Clin 25: 75-90.

2. McMahon DJ, Schwab CW, Kauder D (1996) Comorbidity and the eldery trauma patient. World J Surg 20: 1113-1119.

3. Sohal RS, Weindruch R (1996) Oxidative stress, caloric restriction and aging. Science 273: 59-63.

4. Rooke GA, Robinson BJ (1997) Cardiovascular and autonomic nervous system aging. Problems in Anesthesia 9(4): 482-497.

5. Novak-Jankovic V (2017) Highlights of anaesthesia for elderly patients. Sarajevo anestezija forum 5: 29-41.

\section{ISSN: 2574-1241}

DOI: 10.26717/BJSTR.2018.06.001413

Iztok Potocnik. Biomed J Sci \& Tech Res

(C) (i) This work is licensed under Creative

Submission Link: https://biomedres.us/submit-manuscript.php
6. Bode RH, Lewis KP, Zarich SW, Pierce ET, Roberts M, et al. (1996) Cardiac outcome after peripheral vascular surgery. Comparison of general and regional anaesthesia. Anesthesiology 84: 3-13.

7. Ho AM, Lim HS, Yim AP, Karmakar MK, Lee TW (2002) The resolution of ST segment depressions after high right thoracic paravertebral block during general anesthesia. Anesth Analg 5(1): 227-228.

8. Richardson J, Sabanathan S, Jones J, Shah RD, Cheema S, et al. (1999) A prospective, randomized comparison of preoperative and continuous balanced epidural or paravertebral bupivacaine on post-thoracotomy pain, pulmonary function and stress responses. Brit J Anaesth 83(3): 387-392.

9. Veering B (2007) Regional anesthesia in the elderly, In: Hadzic A (Eds.). Textbook of regional anesthesia and acute pain management. McGraw Hill Medical, New York, Chicago, San Francisco, USA, PP. 787-798.

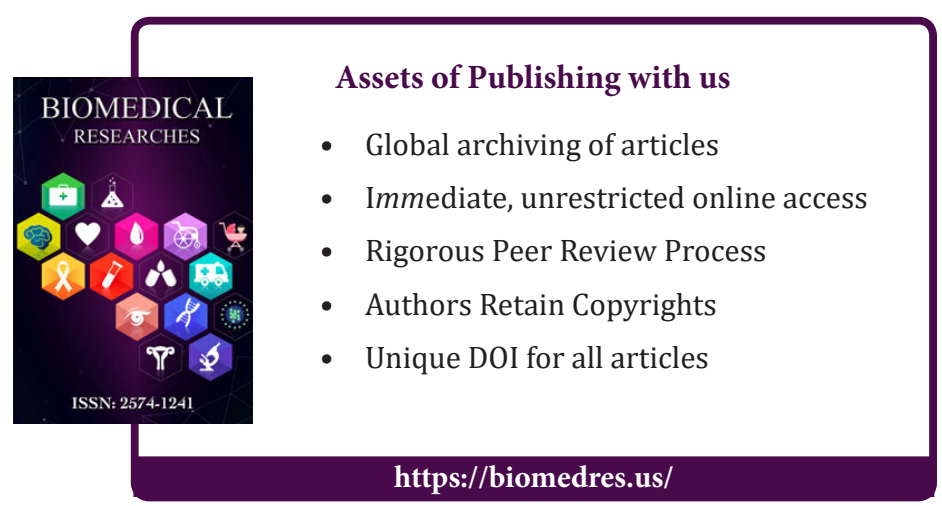

